\title{
Thermodynamics of Cottrell atmospheres tested by atomistic simulations
}

\author{
Y. Mishin ${ }^{1,3}$ and J. W. Cahn ${ }^{2 \dagger}$ \\ ${ }^{1}$ Department of Physics and Astronomy, George Mason University, MSN 3F3, \\ Fairfax, VA 22030, USA \\ 2 Materials Science and Engineering Division, \\ National Institute of Standards and Technology, Gaithersburg, MD 20899, USA \\ 3 Corresponding author. E-mail address: ymishin@gmu.edu (Y. Mishin)
}

July 1, 2016

\begin{abstract}
Solute atoms can segregate to elastically deformed lattice regions around a dislocation and form an equilibrium distribution called the Cottrell atmosphere. We compare two approaches to describe Cottrell atmospheres. In the Eshelby theory, the solid solution is represented by a composite material obtained by insertion of misfitting elastic spheres (solute atoms) into an elastic matrix (solvent). The theory proposed by Larché and Cahn (LC) treats the solution as an elastic continuum and describes elasto-chemical equilibrium using the concept of open-system elastic coefficients. The two theories are based on significantly different concepts and diverge in some of their predictions, particularly regarding the existence of screening of dislocation stress fields by atmospheres. To evaluate predictive capabilities of the two theories, we perform atomistic computer simulations of $\mathrm{Al}$ segregation on a dislocation in Ni. The results confirm the existence of hydrostatic stress screening in good agreement with the LC theory. The composition field is also in much better agreement with the LC prediction than with the Eshelby theory. However, the simulations confirm the logarithmic divergence of the total amount of solute segregation as expected from the Eshelby theory, whereas the LC theory predicts the total segregation to be zero. Several other aspects of the two theories are analyzed. Possible non-linear extensions of the LC theory are outlined.
\end{abstract}

Keywords: Dislocation; Cottrell atmosphere; atomistic simulation; thermodynamics of solid solutions

\footnotetext{
${ }^{\dagger}$ Deceased on March 14, 2016
} 


\section{Introduction}

It is well-known that many solutes interact with elastic strain fields of dislocations and segregate in and around the dislocation core [1]. This segregation, often called the Cottrell atmosphere [1-4], can strongly impact the mobility of dislocations and thus influence mechanical properties of materials. It is important to understand the thermodynamic nature of dislocation atmospheres and be able to predict the amount of segregation and its effect on the dislocation stress field.

As a simple example, consider an edge dislocation with a Burger vector $\mathbf{b}$ in an elastically isotropic solid. The distribution of the pressure $p=-\left(\sigma_{11}+\sigma_{22}+\sigma_{33}\right) / 3\left(\sigma_{i j}\right.$ being the stress tensor) around the dislocation is given by [1]

$$
p=\frac{G b(1+\nu) \sin \theta}{3 \pi(1-\nu) r},
$$

where $G$ is the shear modulus, $\nu$ is Poisson's ratio, $b=|\mathbf{b}|$ and $r$ and $\theta$ are the radius and polar angle, respectively. Cottrell [2-4] suggested that the interaction energy of a solute atom with the dislocation stress field is $U=p \Delta V$, where

$$
\Delta V=v_{s}-v_{a}
$$

Here, $v_{s}$ and $v_{a}$ are the atomic volumes of the solute and solvent, respectively, in their chemically pure unstressed states. This energy represents the reversible work of insertion of a single solute atom in the solvent matrix. For a dilute solution, Cottrell [2-4] obtained the composition field

$$
\frac{c}{c_{0}}=\exp \left(-\frac{p \Delta V}{k_{B} T}\right),
$$

where $c$ is the atomic fraction of the solute atoms near the dislocation, $c_{0}$ is the alloy composition far away from the dislocation, $T$ is temperature and $k_{B}$ is Boltzmann's constant. Thus, oversized $(\Delta V>0)$ substitutional atoms tend to gather in dilated regions $(p<0)$ while undersized $(\Delta V<0)$ in compressed regions $(p>0)$. Cottrell [2-4] suggested that the formation of an atmosphere relieves the hydrostatic stresses $\left(\sigma_{11}+\sigma_{22}+\sigma_{33}\right) / 3$ around the dislocation, and that it is this relief of stress that causes the formation of the atmosphere [3].

Later, Eshelby $[5,6]$ developed a composite model of solid solutions in which the solute atoms were modeled by misfitting elastic spheres embedded in a matrix representing the solvent. This model relies on the small strain approximation, isotropic linear elasticity and the assumption that the inclusions have the same elastic moduli as the matrix. Since the stress field of a dilatation center is pure shear, there is no direct interaction between the inclusions. Consequently, the pressure in the matrix remains, to the leading order, the same as in the pure solvent [1]. In application to dislocation atmospheres, the theory predicts that the composition field is given by the same Eq.(3) as in the Cottrell theory. Furthermore, although the calculations $[5,6]$ were very accurate (within the assumptions of his model) and took full account of the image forces, several terms canceled out and $\Delta V$ ended up being equal to the same difference (2) between the stress-free atomic volumes. 
In this theory, $p$ in Eq.(3) has the meaning of the pressure in the matrix in the absence of the atmosphere. As noted by Hirth $[1,7,8]$, in the standard Eshelby theory there is no screening of the dislocation pressure field by the atmosphere.

In regions far away from the dislocation core where the stress is small enough that $p \Delta V \ll k_{B} T$, one can expand Eq.(3):

$$
\frac{c}{c_{0}}=1-\frac{p \Delta V}{k_{B} T}+\frac{1}{2}\left[\frac{p \Delta V}{k_{B} T}\right]^{2}+\ldots
$$

The total number of solute atoms segregated per unit length of the dislocation line between the radii $r_{0}$ (core radius) and $R$ is

$$
\Gamma=\frac{1}{\omega} \int_{0}^{2 \pi} d \theta \int_{r_{0}}^{R}\left(c-c_{0}\right) r d r
$$

$\omega$ being the atomic volume of the solution. Inserting the expansion (4), the first term integrates to zero while the second gives

$$
\Gamma=\frac{\pi c_{0}}{2 \omega}\left[\frac{G b(1+\nu) \Delta V}{3 \pi(1-\nu) k_{B} T}\right]^{2} \ln \frac{R}{r_{0}} .
$$

It follows that the total segregation is a second order effect, but it still diverges as $R \rightarrow \infty$. It has been shown that if further terms of the series (4) are included, the logarithmic term (6) still remains the dominant contribution [1]. The entire atmosphere contains an infinite amount of the solute.

On the other hand, Larché and Cahn (LC) [9-12] developed a linearized theory of elasto-chemical equilibrium in solid solutions and applied it to dislocation atmospheres $[9,12]$. The LC theory treats the solid solution as an elastic continuum, which can be thought of as obtained by coarse-graining (homogenization) over atomic groups containing a sufficient number of atoms to describe the solution by smooth functions. The LC theory $[9,12]$ predicts that the stress field in the presence of an atmosphere can be obtained by replacing the ordinary elastic coefficients by so-called open-system elastic coefficients. The material in the atmosphere is elastically more compliant than it would be in a closed system. This leads to a reduction in the magnitude of pressure and thus partial screening (relief) of the dislocation stress field, just as predicted by Cottrell $[3,4]$. Knowing the stress field, the composition field in the atmosphere can be computed in the linear approximation. The theory is thermodynamically self-consistent (the elastic and chemical equilibrium problems are fully coupled) and requires thermodynamic input that can be provided by experiment, a CALPHAD assessment [13] or atomistic computer simulations.

This brief overview shows that the existing theories of atmospheres differ in several aspects, particularly regarding the stress screening. The latter question obviously presents significant interest: if the formation of atmospheres weakens elastic interactions between dislocations, then this effect should be taken into account in deformation theories for alloys. The stress screening and other properties of dislocation atmospheres have been the 
subject of a recent debate in the literature $[7,8,14,15]$. The debate was primarily focused on similarities and differences between the Eshelby $[5,6]$ and $\mathrm{LC}[9,12]$ theories. The difficulty in comparing the two theories is in the disparity of certain basic concepts underlying the theories. Each theory is correct within the framework of its basic assumptions and approximations, but the two frameworks are essentially different. Attempts to assess the validity one theory from the standpoint of the other $[7,8,14-16]$ prove ineffective. The only meaningful approach is to identify a set of testable predictions of each theory and compare them with experiments or atomistic simulations. Experimental tests that would provide definitive answers appear unfeasible given the limited capabilities of existing experimental techniques in measuring local stresses and compositions in atmospheres on a quantitative level. Atomistic simulations offer a more viable option at the present time. Such simulations do not rely on any theories, assumptions or approximations other than a model of atomic interactions, which for some systems can be very realistic. This can provide a suitable testing ground for the two theories.

For example, the conclusion of the Eshelby theory $[5,6]$ that the hydrostatic stress in the matrix is unaltered (to the leading order) by the atmosphere is correct within the Eshelby theory but cannot agree or disagree with the LC theory $[9,12]$. The latter does not deal with any matrix or inclusions since the stress appearing in this theory is averaged over volumes containing multiple solute atoms. On the other hand, a recent paper [16] asserts that the hydrostatic self-stress existing inside the elastic inclusions must be excluded when computing the stress dependence of the solute concentration. This assertion suggests that the space-averaged stress that could be compared with the LC theory $[9,12]$ coincides with the matrix stress, and as such, must remain unaltered by the atmosphere. This conclusion contradicts the LC theory $[9,12]$ and the issue can be resolved by computing the stress field in atomistic simulations. Similarly, the composition field of an atmosphere can be computed by atomistic simulations and compared with predictions of both theories. In the Eshelby theory, the composition field will be given by Eqs.(2) and (3), whereas the LC theory makes a different prediction discussed below.

The goal of this work is to perform atomistic computer simulations of an equilibrium atmosphere of a particular dislocation and compare the stress and composition fields with predictions of the two theories. In Sec. 2 we review the LC theory [9, 12] to the extent that is required for this work. The simulation methodology is presented in Sec. 3 followed by simulation results and their interpretation in Sec. 4 . In Sec. 5 we summarize the work and formulate conclusions.

\section{The Larché-Cahn theory}

Larché and Cahn [9-12] developed a rigorous thermodynamic theory of mechanical and chemical equilibrium in and between multicomponent solid phases by generalizing Gibbs' approach [17] that was primarily focused on fluid systems. The equilibrium equations were derived from the minimum energy condition at a fixed entropy. Thermodynamic properties of each solid phase were described by a postulated fundamental equation expressing its energy as a function of entropy, chemical composition and deformation gradient. The 
solution of the extremum problem gave thermodynamic equilibrium conditions for heterogeneous systems and for internal equilibria inside individual phases containing sources of elastic stress. The LC equations represent a complex non-linear problem that cannot be easily solved analytically. To simplify the calculations, a linearization of the equations was proposed $[9,10,12]$ under the assumptions of relatively small stresses and small compositional variations. This linearization enabled Larché and Cahn to decouple the equations in two parts: (i) a linear elasticity problem that can be solved for the stress field, and (ii) a chemical equilibrium problem in a given stress field. The key to this decoupling was the introduction of the concept of open-system elastic coefficients (compliances and stiffnesses), which are different from the ordinary, closed-system elastic coefficients both conceptually and numerically.

For a binary substitutional solid solution, the elasticity problem becomes (summation over repeated indices is implied)

$$
\varepsilon_{i j}=S_{i j k l}^{*} \sigma_{k l}
$$

where $\sigma_{k l}$ and $\varepsilon_{i j}$ are the local stress and small strain tensors, respectively, and

$$
S_{i j k l}^{*} \equiv S_{i j k l}^{0}+\omega\left(\frac{\partial M}{\partial c}\right)_{0}^{-1}\left(\frac{\partial \varepsilon_{i j}^{c}}{\partial c}\right)_{0}\left(\frac{\partial \varepsilon_{k l}^{c}}{\partial c}\right)_{0}
$$

is the tensor of open-system compliances $[9,10,12]$. In Eq.(8), $S_{i j k l}^{0}$ is the tensor of ordinary compliances, $\omega$ is the volume per atom in the reference state of strain, $M$ is the diffusion potential of the solute relative to the solvent, and $\varepsilon_{i j}^{c}$ is the stress-free (compositional) strain. The subscript and superscript 0 indicate the quantities evaluated in the stress-free solid solution far away from dislocations and other sources of stress. This stress-free solution is taken as the reference state of strain. Because $S_{i j k l}^{*}$ does not vary with the local composition $c$ and depends only on the imposed diffusion potential $M$, Eq.(7) is a standard Hooke's law that leads to a linear elasticity problem that can be solved by well-established methods. In fact, in many cases an existing solution can utilized by simply replacing the closed-system elastic coefficients by open-system coefficients. Knowing the stress field $\sigma_{k l}(\mathbf{r})$, the composition field $c(\mathbf{r})$ can be found from the equation

$$
c(\mathbf{r})-c_{0}=\omega\left(\frac{\partial M}{\partial c}\right)_{0}^{-1}\left(\frac{\partial \varepsilon_{k l}^{c}}{\partial c}\right)_{0} \sigma_{k l},
$$

$\mathbf{r}$ being the position vector relative to the dislocation core.

In many cases, for example in cubic solids, the compositional strain $\varepsilon_{i j}^{c}$ is isotropic. Consequently,

$$
\left(\frac{\partial \varepsilon_{i j}^{c}}{\partial c}\right)_{0}=\alpha \delta_{i j}
$$

where $\delta_{i j}$ is Kronecker's symbol and the compositional strain coefficient $\alpha$ is a function of the far-field composition $c_{0}$ but not the local composition $c$. Switching to two-index notations for elastic compliances [18] we then have

$$
S_{i j}^{*}=S_{i j}^{0}+\xi, \quad i, j=1,2,3
$$




$$
S_{i j}^{*}=S_{i j}^{0}, \quad i \text { and } / \text { or } j>3
$$

where

$$
\xi=\alpha^{2} \omega\left(\frac{\partial M}{\partial c}\right)_{0}^{-1}
$$

is a thermodynamic factor. Thus, for cubic crystals $S_{11}^{*}$ and $S_{12}^{*}$ are shifted relative to their closed-system counterparts by the same amount $\xi$, whereas $S_{44}^{*}$ remains unchanged. The equation for the composition field (9) becomes

$$
c(\mathbf{r})-c_{0}=-3 \omega \alpha\left(\frac{\partial M}{\partial c}\right)_{0}^{-1} p .
$$

In application to a dislocation atmosphere, the linearized LC method $[9,12]$ consists in finding the dislocation stress field $p(\mathbf{r})$ by replacing $S_{i j}^{0}$ by $S_{i j}^{*}$ in a known linear elasticity solution, followed by calculation of the composition field $c(\mathbf{r})$ from Eq.(14).

Consider the particular case of an elastically isotropic solid. In the absence of an atmosphere, the pressure field of an edge dislocation is given by Eq.(1). Using this equation, the pressure field in the atmosphere becomes $[9,12]$

$$
p=\frac{G b\left(1+\nu^{*}\right) \sin \theta}{3 \pi\left(1-\nu^{*}\right) r}=\frac{G b(1+\nu) \sin \theta}{3 \pi[1-\nu+4(1+\nu) G \xi] r},
$$

where $\nu^{*}$ is the open-system Poisson's ratio. (Note that the shear modulus remains unchanged.) Because for thermodynamically stable solutions $(\partial M / \partial c)>0$, we always have $\xi>0$ and the magnitude of pressure is always reduced relative to the pre-existing pressure by a factor of

$$
\frac{1-\nu}{1-\nu+4(1+\nu) G \xi}<1
$$

Since in this theory the composition is linear in pressure, the total segregated amount $\Gamma$ computed from Eq.(5) is zero.

It is interesting to compare the LC Eq.(14) with Eq.(3) of the Eshelby theory in the dilute solution limit. Linearization of the latter equation gives

$$
c-c_{0}=-\frac{c_{0} p \Delta V}{k_{B} T} .
$$

On the other hand, for a dilute solution $(\partial M / \partial c)_{0}=k_{B} T / c_{0}$ and Eq.(14) becomes

$$
c-c_{0}=-\frac{c_{0} p V^{*}}{k_{B} T}
$$

where

$$
V^{*}=3 \omega \alpha
$$

can be called the "effective volume" of insertion of a solute atom. In the small-strain approximation, $V^{*}$ is the difference between the partial molar volumes of the solute and solvent. Comparing the two equations, we observe that the "effective volume" appearing in the LC theory is the counterpart of $\Delta V$ of the Eshelby theory. A comparison between the two volumes will be discussed later. 


\section{Methodology}

As a model system we chose the FCC Ni-Al solid solution known as the gamma-phase [19]. The lattice parameter mismatch between $\mathrm{Ni}$ and $\mathrm{Al}$ is large (about $15 \%$ ) suggesting a strong segregation trend. Atomic interactions in the Ni-Al system were described by an embedded-atom potential [20] that accurately reproduces thermodynamic properties of the $\mathrm{Ni}-\mathrm{Al}$ system. In particular, the potential predicts the $\mathrm{Ni}$-rich part of the $\mathrm{Ni}-\mathrm{Al}$ phase diagram in reasonable agreement with experiment. The simulations were carried out at a fixed temperature of $700 \mathrm{~K}$. At this temperature, the solubility limit of Al in the gammaphase predicted by this potential is about $13.9 \% \mathrm{Al}$ (mole fraction). This is higher than the experimental solubility limit, but this deviation is not critical for the testing purposes set in this work. Two types of simulation were performed: simulations in a small system for computing the elastic constants and thermodynamic parameters required for this work, and simulations in a larger system to study a dislocation atmosphere.

For computing thermodynamic properties and elastic constants, we used a periodic block of FCC lattice containing $N_{a}=2034$ atoms with approximately cubic dimensions. The lattice orientation relative to the block edges was $[001] \| x_{1},[110]|| x_{2}$, [110] ||$x_{3}$. Semigrand canonical Monte Carlo simulations [21] were implemented using the parallel Monte Carlo code ParaGrandMC [22] at $700 \mathrm{~K}$ for a set of diffusion potentials $M$ of Al with respect to Ni. In the semi-grand canonical ensemble, temperature $T$ and diffusion potential $M$ are fixed while the system dimensions and chemical composition can vary, mimicking an open system. The trial moves of the Monte Carlo process included displacements of randomly selected atoms by a random amount in a random direction with simultaneous random re-assignment of the chemical species of the chosen atom to either $\mathrm{Ni}$ or Al. $N_{a}$ trial moves constitute one Monte Carlo step. After each Monte Carlo step, the dimensions of the simulation block in the $x_{1}, x_{2}$ and $x_{3}$ Cartesian directions were changed independently by small random amounts with corresponding re-scaling of atomic coordinates. The fluctuations of the system dimensions ensured zero normal stresses $\sigma_{11}, \sigma_{22}$ and $\sigma_{33}$. The trial moves were accepted or rejected by the Metropolis algorithm [21]. Namely, a trial move was accepted with the probability of $\exp \left(-\Phi / k_{B} T\right)$ if $\Phi>0$ and unconditionally if $\Phi \leq 0$, where $[23-25]$

$$
\Phi \equiv \Delta E \mp M \pm \frac{3}{2} k_{B} T \ln \frac{m_{\mathrm{Ni}}}{m_{\mathrm{Al}}}
$$

Here, $M$ is the diffusion potential of the reservoir and $m_{\mathrm{Ni}}$ and $m_{\mathrm{Al}}$ are the atomic masses and $\Delta E$ is the total energy change resulting from the move. The positive sign applies when $\mathrm{Ni}$ is replaced by $\mathrm{Al}$ and negative when $\mathrm{Al}$ is replaced by $\mathrm{Ni}$. The mass term comes from integration of the state probability over linear momenta of atoms, giving a pre-exponential factor proportional to the product of masses of all atoms to the power of $3 / 2$. In the probability ratio of two configurations, all masses cancel out except for the masses of the atom whose species changes, producing a pre-exponential factor of either $\left(m_{\mathrm{Ni}} / m_{\mathrm{Al}}\right)^{3 / 2}$ or $\left(m_{\mathrm{Al}} / m_{\mathrm{Ni}}\right)^{3 / 2}$. For each $M$, the simulation started with pure Ni and continued until thermodynamic equilibrium was reached. The equilibrium state was verified by computing the non-Gaussian parameters of statistical distributions. The equilibration usually required about $2 \times 10^{4}$ Monte Carlo steps (verified by small non-Gaussian param- 
eters of statistical distributions). After the equilibration, a production run was performed comprising about $10^{6}$ Monte Carlo steps. At each Monte Carlo step, we recorded the current dimensions $L_{1}, L_{2}$ and $L_{3}$ of the simulation block and its chemical composition $c$. The block dimensions were converted to the fluctuating strains $\varepsilon_{i i}=\left(L_{i}-\bar{L}_{i}\right) / \bar{L}_{i}$, where the bar denotes the ensemble average.

It should be emphasized that the Monte Carlo sampling of the equilibrium state automatically includes interatomic bonding, atomic vibrations (including anharmonicity), the configurational degrees of freedom (permutations of atoms) and fluctuations of the system dimensions.

It can be shown [26] that elastic compliances are proportional to the covariances of the strains $\overline{\varepsilon_{i i} \varepsilon_{j j}}$ :

$$
\tilde{S}_{i j}^{*}=\frac{\bar{V}}{k_{B} T} \overline{\varepsilon_{i i} \varepsilon_{j j}}, \quad i, j=1,2,3,
$$

where $\bar{V}$ is the average volume of the system. The asterisk indicates that these compliances are open-system type and the tilde is a reminder that they are computed for a lattice orientation different from the cubic axes. The $3 \times 3$ matrix of elastic compliances obtained from the fluctuations was inverted to obtain the matrix of open-system elastic constants $\tilde{c}_{i j}^{*}(i, j=1,2,3)$. The open-system elastic constants in the cubic system $\left(c_{11}^{*}, c_{12}^{*}\right.$ and $\left.c_{44}^{*}\right)$ were then obtained by an appropriate rotation of the coordinate axes, see [27] for details.

Knowing $c_{i j}^{*}$, the closed-system elastic constants $c_{i j}$ were recovered by converting $c_{i j}^{*}$ to the compliances $S_{i j}^{*}$ and utilizing Eqs.(11) and (12) to find $S_{i j}^{0}$ and thus $c_{i j}$. This calculation required the knowledge of the thermodynamic factor $\xi$ given by Eq.(13). The diffusion potential $M$ and the cubic lattice parameter $a$ were known from the simulations as functions of composition. The derivatives of these functions gave the required ingredients of $\xi:(\partial M / \partial c)$ and $\alpha=(\partial a / \partial c) / a$.

The foregoing procedure for computing the elastic coefficients $c_{i j}^{*}$ and $c_{i j}$ was validated [27] by additional, computationally demanding simulations implementing the canonical ensemble, in which the trial Monte Carlo moves included swaps of randomly selected pairs of atoms. This ensemble simulates a closed system and permits a direct calculation of $c_{i j}$ from the shape fluctuations. The results were found to be in close agreement with the calculations in which $c_{i j}$ was extracted from $c_{i j}^{*}$ through the thermodynamic factor $\xi$. This agreement demonstrates the internal consistency of the LC definition of the open-system elastic coefficients.

The dislocation chosen for this study was edge with the Burgers vector $\mathbf{b}=[100]$. This dislocation is different from the normal (1/2)[110] dislocations in FCC metals but is wellsuited for testing purposes. This dislocation has a longer Burgers vector $(b \approx 0.352 \mathrm{~nm})$ producing larger stresses and has a compact core (there are no stable stacking faults on cubic planes) so it can be better described by elasticity theory solutions than dislocations with a dissociated core. We first created a rectangular $30 \times 30 \times 3.4 \mathrm{~nm}$ simulation block of FCC Ni with edges aligned with coordinate axes $x_{i}$. The lattice orientation was $[100] \| x_{1}$, $[010]\left\|x_{2},[001]\right\| x_{3}$ with periodic boundary conditions in all Cartesian directions. A zeropressure semi-grand canonical Monte Carlo simulation was implemented on this system at $T=700 \mathrm{~K}$ and $M=-0.73 \mathrm{eV}$ until equilibrium was reached. The equilibrium chemical composition was found to be $5.43 \% \mathrm{Al}$, i.e., $c_{0}=0.0543$. One of the saved configurations 
with this composition was chosen for the subsequent study. In this configuration, the periodic boundary conditions in $x_{1}$ and $x_{2}$ were canceled and a dislocation was created at $x_{1}=x_{2}=0$ by displacing all atoms from their positions according to the displacement field of an edge dislocation given by the anisotropic elasticity solution [28] with open-system elastic constants $c_{i j}^{*}$ computed for this composition. The dislocation line was parallel to the shorter $\left(x_{3}\right)$ direction of the block. A cylindrical region with the diameter of $26.6 \mathrm{~nm}$ (162,496 atoms) centered at the dislocation line was then carved out of the block. Since the boundary condition along the $x_{3}$ axis remained periodic, this effectively created an infinitely long cylinder with the dislocation line coinciding with its axis (Fig. 1).

At the next step, all atoms located in a $1.5 \mathrm{~nm}$ thick surface layer of the cylinder were fixed in their positions, whereas all other atoms remained free (Fig. 1a). Finally, a semigrand canonical Monte Carlo simulation was executed on the free atoms at $T=700 \mathrm{~K}$ and $M=-0.73 \mathrm{eV}$. The trial moves of the simulation included local atomic displacements and changes of chemical identities of atoms but not the re-scaling of atomic coordinates. Note that the fixed atoms of the surface layer continued to impose traction on the free atoms. $10^{6}$ Monte Carlo steps were found to be more than sufficient to bring the system to thermodynamic equilibrium, and thus form an equilibrium dislocation atmosphere, and to generate over 600 uncorrelated snapshots from the equilibrium state. During the simulations, the fixed atoms remained at nearly zero stress and maintained the far-field composition $c_{0}$ (Fig. 1b).

At the post-processing stage, the coarse-grained stress and composition fields were computed in each snapshot and averaged over all snapshots. For the coarse-graining, a $b \times b$ square mesh was superimposed on the $\left(x_{1}, x_{2}\right)$ projection of the simulation block and the chemical composition and stress were averaged over the atoms projected into each square unit from the $x_{3}$ dimension (Fig. 1c). The stress tensor was computed by the standard virial expression including the thermal term.

To provide a baseline for comparison, similar simulations were performed for the same dislocation in pure Ni. In this case, the Monte Carlo moves did not include switching the chemical identities of atoms and the elastic constants were $c_{i j}$ of pure Ni at $700 \mathrm{~K}$.

\section{Results and discussion}

The elastic constants obtained for pure $\mathrm{Ni}$ and the $\mathrm{Ni}-5.43 \% \mathrm{Al}$ alloy at $700 \mathrm{~K}$ are listed in Table 1. The estimated error bar of this calculation method is \pm 1 GPa [27]. The table shows that the addition of $\mathrm{Al}$ to $\mathrm{Ni}$ has only a small effect on the ordinary (closed-system) elastic constants. However, the open-system elastic constants of the alloy are about 16 GPa $(10 \%)$ lower than the ordinary elastic constants. Due to this chemical softening effect, we can already anticipate a reduction in pressure around the dislocation.

The pressure field around the dislocation in pure $\mathrm{Ni}$ is shown in Fig. 2 in comparison with predictions of the anisotropic elasticity theory. In Fig. 2(b), the pressure is plotted as a function of distance $r$ from the core when moving in the vertical direction (the $x_{2}$ axis) passing through the dislocation core (red arrow in Fig. 1(c)). The points represent the pressures averaged over $b \times b$ cells. The agreement between the elasticity theory and 
the simulations is excellent except in the dislocation core region where linear elasticity is inapplicable. To highlight this agreement, the pressure is re-plotted in Fig. 2(c) against $1 / r$. We again observe excellent agreement near the origin of the plot (far field) and significant deviations in the core region (top left and bottom right corners of the plot). This close agreement demonstrates the accuracy of the present simulation setup and of our calculations of elastic constants and stresses.

Fig. 3 compares the simulations with elasticity theory predictions for the $\mathrm{Ni}-5.43 \% \mathrm{Al}$ alloy. The agreement is again very good, except of course in the core region. This agreement shows that the LC theory with open-system elastic coefficients accurately describes the dislocation stress field. For comparison, the star points show the pressure field in pure $\mathrm{Ni}$ (replotted from Fig. 2(b)). The Eshelby theory predicts $[5,6,16]$ that the pressure field in the atmosphere should be the same as in pure Ni (matrix). In fact, the magnitude of the Ni pressure systematically overestimates the simulation results. The deviation is relatively small but undoubtedly indicates the existence of partial screening.

The composition field around the dislocation is displayed in Fig. 4(a). As expected, the regions of depletion and enrichment in $\mathrm{Al}$ correlate with the regions of compression and tension, respectively. In Fig. 4(b), the composition averaged over $b \times b$ cells is plotted as a function of distance $r$ from the dislocation core moving in the vertical direction. The points represent simulation results. As evident from Fig. 4(b), the simulations accurately match the prediction of the LC theory despite the statistical scatter of the data points. (Since the Al concentration is relatively small, the statistical scatter is significant and could not be reduced further with computational resources available to us.) For comparison, we plot the composition curves predicted by the Cottrell and Eshelby theory [1-8]. The curves were computed from the equation

$$
c=\frac{c_{0}}{c_{0}+\left(1-c_{0}\right) \exp \left(\frac{p \Delta V}{k_{B} T}\right)},
$$

generalizing Eq.(3) to non-dilute compositions [1, 16]. The curves calculated directly from Eq.(3) look very similar (not shown) and lead to the same conclusion. Namely, the Cottrell and Eshelby theory strongly overestimates the solute enrichment and depletion in the respective regions around the dislocation.

The main source of this deviation is the fact that Eq.(2) identifies the volume effect of solution with the difference $\Delta V$ between the stress-free atomic volumes of the components. The LC counterpart of $\Delta V$ is the effective volume $V^{*}$ given by Eq.(19). Replacement of $\Delta V$ by $V^{*}$ gives Eq.(18) that matches the simulation results much more closely than Eqs.(2) and (3). Fig. 5(a) demonstrates that for the Ni-Al system, $\Delta V$ overestimates $V^{*}$ by approximately a factor of four, causing the large over-predication of the enrichment/depletion. To demonstrate that $\mathrm{Ni}-\mathrm{Al}$ is not a rare case, we quote the results of similar calculations for the $\mathrm{Cu}-\mathrm{Ag}$ system described by an embedded-atom potential [29]. The potential accurately predicts many physical and thermodynamic properties of $\mathrm{Cu}, \mathrm{Ag}$ and $\mathrm{Cu}-\mathrm{Ag}$ solutions, and in particular predicts the phase diagram in reasonable agreement with experiment. In this system, $\Delta V$ is close to that in the $\mathrm{Ni}-\mathrm{Al}$ system. We find that $\Delta V$ underestimates $V^{*}$ in the Cu-rich solid solution (Fig. 5(b)) and over- 
estimates in the Ag-rich solid solution (Fig. 5(c)) [30]. At the temperature of $1000 \mathrm{~K}$, the difference between $\Delta V$ and $V^{*}$ is 20 to $30 \%$, which is not as large as in Ni-Al at $700 \mathrm{~K}$ but still significant. That the difference is not as large is not surprising given the chemical similarity of $\mathrm{Cu}$ and $\mathrm{Ag}$ and disparity of $\mathrm{Ni}$ and $\mathrm{Al}$. It should also be noted that the difference between $\Delta V$ and $V^{*}$ increases with decreasing temperature. This analysis illustrates the obvious fact that predictions of the volume effect of solution solely on the basis of atomic volumes of the end members is a crude approximation. The LC theory incorporates both atomic size and chemical effects, leading to much more accurate predictions of the composition field in the dislocation atmosphere.

As indicated in Secs. 1 and 2, the two theories give different answers for the total number $\Gamma$ of excess solute atoms per unit dislocation line. According to the linear LC theory $\Gamma$ is identically zero, whereas the Cottrell-Eshelby theory predicts that $\Gamma$ is small (second order effect) but eventually diverges as $\ln R$ as $R \rightarrow \infty$. In Fig. 6(a), we plot $\Gamma$ obtained by the simulations as a function of the radius $R$. The first thing to note is that $\Gamma$ is very small. Even for $R=10 \mathrm{~nm}$, the atmosphere contains only two extra $\mathrm{Al}$ atoms per $1 \mathrm{~nm}$ of length. For comparison, the same volume of the Ni-5.43\% Al solid solution contains $1565 \mathrm{Al}$ atoms. Furthermore, the plot of $\Gamma$ versus $\ln R$ is fairly linear (Fig. 6(b)), in agreement with the prediction of the Cottrell-Eshelby theory [1] (Eq.(6)). The failure of the LC theory to predict non-zero $\Gamma$ stems from the linear relation between the local composition and pressure. The correct logarithmic divergence of $\Gamma$ arising in the Cottrell-Eshelby theory is due to the non-linear composition-pressure relation given by Eq.(3).

\section{$5 \quad$ Summary and conclusions}

The Eshelby [5, 6] and Larché-Cahn [9, 12] theories of dislocation atmospheres have been compared with each other and tested against atomistic simulations. One of the points where the theories diverge is the existence of stress relief (screening) by the atmosphere. Such a relief was predicted qualitatively by Cottrell [2-4], not predicted by the Eshelby theory $[5,6]$, predicted quantitatively by the LC theory, and confirmed by the present simulations. The amount of stress relief found by the simulations is in good agreement with the LC theory (Fig. 3).

In the particular $\mathrm{Ni}-\mathrm{Al}$ system studied here, the amount of stress relief is relatively small, although not at all negligible. More systematic investigations are needed to get a fuller idea about the strength of screening in other alloy systems. The answer will evidently depend not only on the atomic size mismatch but also thermodynamic specifics of the system. In particular, it would be interesting to examine a system with a small lattice mismatch $\Delta V$ but strong chemical effects (e.g., Al-Au), in which the atmospheres are largely driven by chemistry. Likewise, the effect of the lattice mismatch alone could be evaluated by studying an unlimited solubility system such as Si-Ge wherein the solid solution is close to ideal. Equations (8) and (13) show that, due to the presence of the compositional strain, the open-system elastic compliances will still be larger than the ordinary ones. This will lead to a stress screening, although the magnitude of the effect 
is likely to be smaller than in the non-deal $\mathrm{Ni}-\mathrm{Al}$ system studied in this work. Another interesting direction of future work would be to study interstitial solid solutions such as $\mathrm{Fe}-\mathrm{C}$ or $\mathrm{Zr}-\mathrm{H}$. The compositional strain can be large and the screening significant. The LC equations remain the same except that the diffusion potential must be replaced by the chemical potential of the interstitial solute.

The stress relief is a consequence and not the "cause" of atmosphere formation as was thought initially [3]. The main thermodynamic driving force for the atmosphere formation is the work $p V^{*}$ done by/on the local pressure $p$ in changing the volume by $V^{*}$ due to the insertion/removal of a solute atom. This insertion/removal does relieve the local pressure $p$, but this is a second order effect. As discussed by Larché and Cahn [9, 12], the atmosphere problem must be solved iteratively: the stress field causes redistribution of the solute atoms, which modifies the local lattice parameter (compositional strain) in the atmosphere, which in turn affects the stress field, and so on until the composition and stress fields become self-consistent [9, 12]. Viewed from this perspective, the Eshelby theory truncates the process after the first iteration, leaving the initial stress (in the absence of an atmosphere) unaltered.

One can imagine situations where the screening of stress fields can be very strong. If the solid solution is metastable and close to a spinodal line or critical point of mixing, then $(\partial M / \partial c)_{0} \rightarrow 0, \xi \rightarrow \infty$, and thus $S_{i j}^{*} \rightarrow 0$. The material then becomes "super-compliant" and the dislocation pressure field can be screened almost completely. Consequences for plastic deformation can be dramatic. Of course, this screening requires diffusion and time to let the atmosphere reach equilibrium. For substitutional solid solutions, the situation is more relevant to creep conditions rather than low-temperature deformation. In interstitial solutions, however, diffusion is usually fast and the screening can be achieved at relatively low temperatures. In some cases, the metastable solution may decompose with the formation of a cylinder of a new phase on the dislocation core.

The proposed hypothetical scenario of nearly complete screening might be worth testing by atomistic simulations. Metal-hydrogen systems are obvious candidates due to the fast formation of atmospheres by mobile hydrogen atoms.

The LC theory accurately predicts the composition field of the dislocation (Fig. 4). By contrast, the Cottrell-Eshelby equations (2) and (3) strongly deviate from the simulation results. This deviation can be traced back to the overestimated volume effect by Eq.(2). This equation is a crude approximation that may only work for some systems that closely follow Vegard's rule. Generally, the effective volume $V^{*}$ of the LC theory is a more accurate predictor of dislocation atmospheres.

On the other hand, the LC prediction of zero total segregation $\Gamma$ is not borne out by the simulations. Although $\Gamma$ is indeed very small (Fig. 6), it increases with the radius $R$ of the cylindrical region and follows the $\ln R$ dependence expected from the Cottrell and Eshelby theories (Fig. 6). This shortcoming is a consequence of the linear character of the LC theory. In a recent paper [14], the linear relation (18) was rewritten in the exponential form

$$
\frac{c}{c_{0}}=\exp \left(-\frac{p V^{*}}{k_{B} T}\right)
$$

similar to Eq.(3). Although this step has a somewhat ad hoc character given that the 
elasticity problem still remains linear, it immediately recovers the $\ln R$ dependence of $\Gamma$ except that $\nu$ and $\Delta V$ in Eq.(6) are replaced by $\nu^{*}$ and $V^{*}$, respectively. A more rigorous approach is offered by the non-linear version of the LC theory proposed for binary alloys [11] and recently generalized to multicomponent systems [27]. In this version, a term linear in $p$ is included in the open-system compliances and the chemical composition equation (18) acquires an additional term quadratic in $p$ (see, e.g., Eqs.(A12) and (A14) in [27]). The elasticity problem becomes non-linear and more complex, but once it is solved, the rest of the calculation is straightforward. The only additional parameter needed for the calculations is the thermodynamic quantity $\left(\partial^{2} M / \partial c^{2}\right)_{0}$, which can be readily computed from the input thermodynamic data. The theory recovers the logarithmic dependence of $\Gamma$ with a pre-logarithmic coefficient different from that in Eq.(6).

As already mentioned, both theories are logically consistent within their assumptions and approximations. A meaningful way to evaluate their utility is to compare their predictions with atomistic simulations. This has been done, and the comparison generally favors the LC theory.

Other criteria include the richness of physical effects captured by a theory and possibilities of its systematic improvement. In this respect, the LC theory also has significant advantages over the Eshelby model. The latter is purely mechanical and does not require any material input other than the elastic constants of the pure solvent and lattice parameters of pure components. The chemical interactions, atomic vibrations, thermodynamic stability of the solution and other physical effects are not included. As mentioned above, the estimate of the volume effect by Eq.(2) is a crude approximation. Extension of the theory to non-dilute solutions is formally possible $[1,16]$ but is unlikely to yield reliable results. (In a random 50:50 solution, even the concepts of a "matrix" and "inclusions" become subjective.) More detailed analyses and critiques of the theory can be found in the literature, e.g. [31]. Although some predictions of the theory are qualitatively correct and can explain a number of properties of atmospheres, the theory is not intended for quantitative predictions.

The LC theory includes all misfit, chemical and vibrational effects, which are incapsulated in the thermodynamic functions and compositional strains required as input. The theory does not rely on the solid solution being dilute, ideal or regular - it can have any complexity and any number of components as long as the relevant thermodynamic data is available. The main limitation of the theory is in its linear character that permits an analytical treatment but becomes less accurate for large concentration gradients. The linear version of the theory can be improved by including quadratic terms [11, 27]. If needed, higher-order terms can also be added by systematically applying the same power expansion procedure as in $[11,27]$. The equations become more complex and must be solved numerically, but predictions of the theory will be increasingly more reliable. Ultimately, it could be easier to solve the original non-linear equations of the theory [9-11] (e.g., Eqs.(10) and (13) in [27]) numerically. Although the beauty of analytical solutions is lost, these more general forms of the theory can describe atmospheres in both stable and metastable solutions, including spinodals and critical points/lines. Interesting phenomena can be studied, such as the nearly full screening mentioned above or sudden collapse of the atmosphere with the formation of a stable second phase on a dislocation in a single-phase 
bulk solution.

The input information required by the LC theory includes the compositional strain, elastic coefficients (open or closed-system type) and diffusion potentials of the components as function of chemical composition and temperature. This information can be provided by thermodynamic databases or atomistic computer simulations. The latter approach has been demonstrated in this work. We envision that in the future, as computational power increases, it should become possible to generate all input to the LC theory by first-principles calculations.

Acknowledgements: This work was presented by the authors at the TMS Annual Meeting and Exhibition in March, 2015. While the paper was in preparation, J.W.C. passed away on March 14, 2016. Y.M. takes responsibility for the final version of the manuscript. This research was supported by the National Institute of Standards and Technology, Material Measurement Laboratory, the Materials Science and Engineering Division.

\section{References}

[1] J. P. Hirth, J. Lothe, Theory of Dislocations, 2 ed., Wiley, New York, 1982.

[2] A. H. Cottrell, Effect of solute atoms on behavior of dislocations, in: Report of a Conference on Strength of Solids, The Physical Society, Lodon, UK, 1948, pp. 30-38.

[3] A. Cottrell, B. A. Bilby, Dislocation theory of yielding and strain aging of iron, Proc. Phys. Soc. London 62 (1949) 49-62.

[4] A. H. Cottrell, Dislocations and plastic flow in crystals, Clarendon Press, Oxford, 1953.

[5] J. D. Eshelby, The determination of the elastic field of an ellipsoidal inclusion, and related problems, Proc. Roy. Soc. London Ser. A-Math. 241 (1957) 376-396.

[6] J. D. Eshelby, The elastic field outside an ellipsoidal inclusion, Proc R Soc London Ser A-Math 252 (1959) 561-569.

[7] J. P. Hirth, On definitions and assumptions in the dislocation theory for solid solutions, Philos. Mag. 94 (2014) 3162-3169.

[8] J. P. Hirth, Response to comments, Philos. Mag. 94 (2014) 3177-3184.

[9] F. Larché, J. W. Cahn, A linear theory of thermochemical equilibrium of solids under stress., Acta Metall. 21 (1973) 1051-1063.

[10] F. C. Larché, J. W. Cahn, Thermodynamic equilibrium of multiphase solids under stress, Acta Metall. 26 (1978) 1579-1589. 
[11] F. C. Larché, J. W. Cahn, A nonlinear theory of thermochemical equilibrium under stress, Acta Metall. 26 (1978) 53-60.

[12] F. C. Larché, J. W. Cahn, The interactions of composition and stress in crystalline solids, Acta Metall. 33 (1985) 331-367.

[13] H. L. Lukas, S. G. Fries, B. Sundman, Computational thermodynamics. The Calphad method., Cambridge University Press, Cambridge, UK, 2007.

[14] J. W. Cahn, Thermodynamic aspects of Cottrell atmospheres, Philos. Mag. 93 (2013) $3741-3746$.

[15] J. W. Cahn, Reprise: partial chemical strain dislocations and their role in pinning dislocations to their atmospheres, Philos. Mag. 94 (2014) 3170-3176.

[16] W. Cai, R. B. Sills, D. M. Barnett, W. D. Nix, Modeling a distribution of point defects as misfitting inclusions in stressed solids, J. Mech. Phys. Solids 66 (2014) $154-171$.

[17] J. W. Gibbs, On the equilibrium of heterogeneous substances, in: The collected works of J. W. Gibbs, volume 1, Yale University Press, New Haven, 1948, pp. 55-349.

[18] J. F. Nye, Physical Properties of Crystals, Clarendon Press, Oxford, 1985.

[19] T. B. Massalski (Ed.), Binary Alloy Phase Diagrams, ASM, Materials Park, OH, 1986.

[20] Y. Mishin, Atomistic modeling of the $\gamma$ and $\gamma^{\prime}$ phases of the Ni-Al system, Acta Mater. 52 (2004) 1451-1467.

[21] D. Frenkel, B. Smit, Understanding molecular simulation: from algorithms to applications, second ed., Academic, San Diego, 2002.

[22] The ParaGrandMC code can be obtained from the NASA Software Catalog. URL: https://software.nasa.gov/software/LAR-18773-1.

[23] J. A. Brown, Y. Mishin, Effect of surface stress on Ni segregatin in (110) NiAl thin films, Phys. Rev. B 69 (2004) 195407.

[24] J. A. Brown, Y. Mishin, Segregation and structural transformations at $\Sigma 3$ grain boundaries in NiAl: A Monte Carlo study, Acta Mater. 53 (2005) 2149-2156.

[25] Y. Mishin, Calculation of the $\gamma / \gamma^{\prime}$ interface free energy in the Ni-Al system by the capillary fluctuation method, Modeling Simul. Mater. Sci. Eng. 22 (2014) 045001.

[26] M. Parrinello, A. Rahman, Strain fluctuations and elastic constants, J. Chem. Phys. 76 (1982) 2662-2666.

[27] Y. Mishin, Calculation of open and closed system elastic coefficients for multicomponent solids, Phys. Rev. B 91 (2015) 224107. 
[28] A. N. Stroh, Dislocations and cracks in anisotropic elasticity, Philos. Mag. 3 (1958) 625-646.

[29] P. L. Williams, Y. Mishin, J. C. Hamilton, An embedded-atom potential for the Cu-Ag system, Modelling Simul. Mater. Sci. Eng. 14 (2006) 817-833.

[30] J. Hickman, Y. Mishin, Disjoining potential and grain boundary premelting in binary alloys, 2016. Submitted to Physical Review B (2016).

[31] J. W. Christian, The theory of transformations in metals and alloys, volume 1, Pergamon Press, Oxford, UK, 2002, pp. 200-208, 269-273. 


\begin{tabular}{|c|c|c|c|c|c|c|}
\hline & \multicolumn{3}{|c|}{ Closed system } & \multicolumn{4}{c|}{ Open system } \\
\hline \hline Elastic constants (GPa) & $c_{11}$ & $c_{12}$ & $c_{44}$ & $c_{11}^{*}$ & $c_{12}^{*}$ & $c_{44}^{*}$ \\
\hline $\mathrm{Ni}-5.43 \% \mathrm{Al}$ & 204.4 & 135.7 & 108.6 & 187.6 & 119.0 & 108.6 \\
\hline $\mathrm{Ni}$ & 207.1 & 132.7 & 106.0 & & & \\
\hline
\end{tabular}

Table 1: Open and closed-system elastic constants of $\mathrm{Ni}$ and the Ni-5.43 \% Al solid solution at the temperature of $700 \mathrm{~K}$. 
(a)

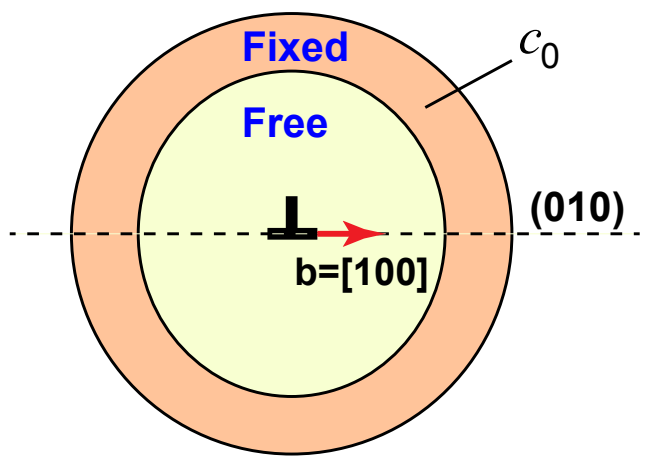

(b)

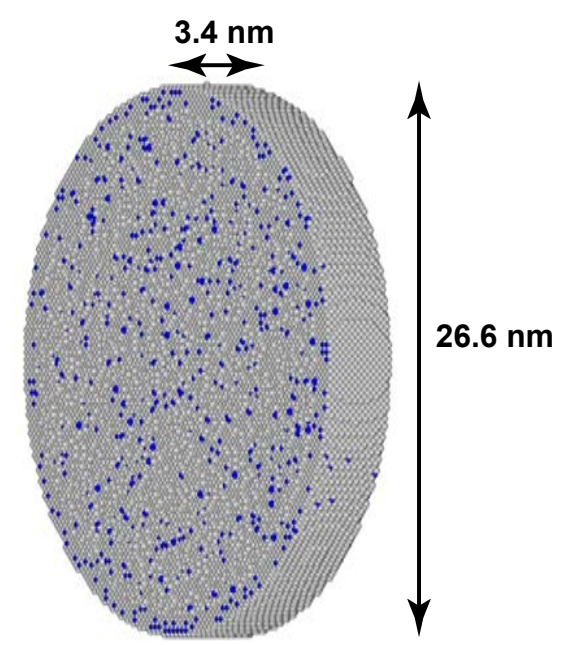

(c)

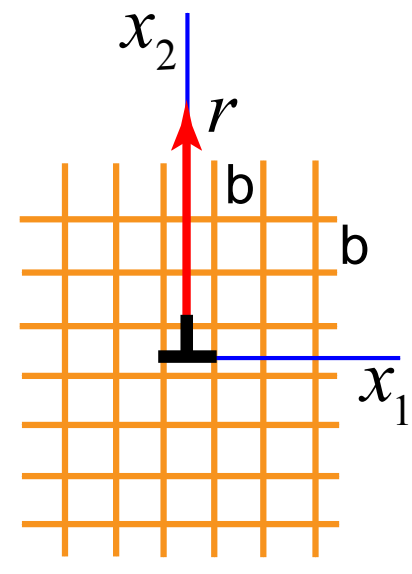

Figure 1: Dislocation studied in this work. (a) Schematic simulation block showing the regions of fixed and fee atoms. (b) Snapshot of Monte Carlo simulations showing Ni (grey spheres) and $\mathrm{Al}$ (blue spheres) atoms. (c) Mesh for the coarse-graining of pressure and chemical composition. 
(a)
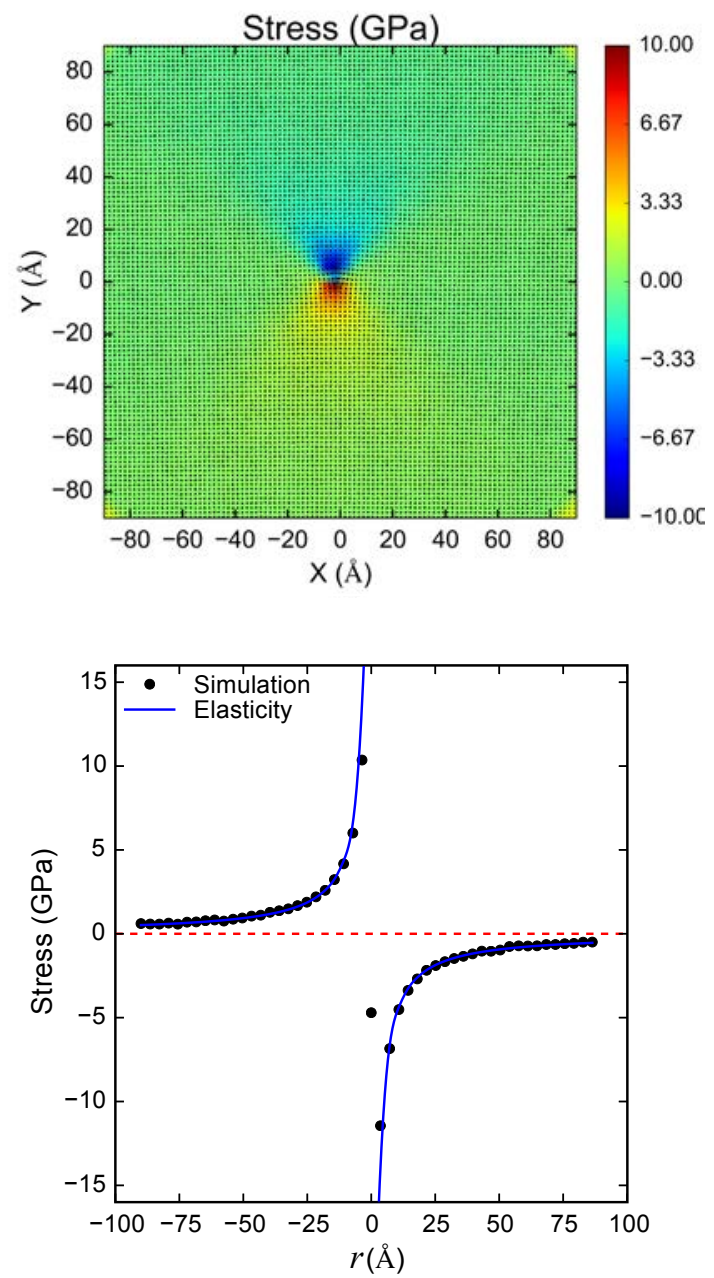

(b)

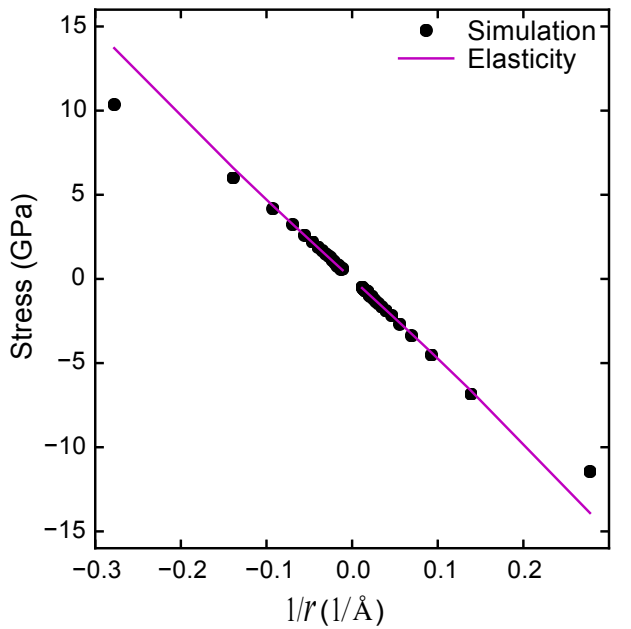

(c)

Figure 2: Hydrostatic stress (negative of pressure) around the dislocation in $\mathrm{Ni}$ at 700 K. (a) Color map of hydrostatic stress showing regions of tension and compression. (b) Hydrostatic stress as a function of distance $r$ in the vertical direction. (c) Hydrostatic stress as a function of inverse distance $1 / r$ in the vertical direction. The points represent simulation results; the curves are predictions of the elasticity theory. 


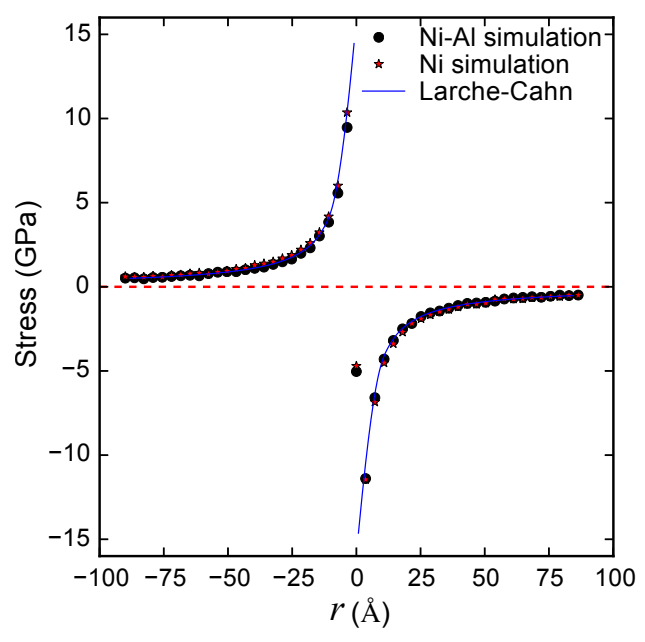

(a)

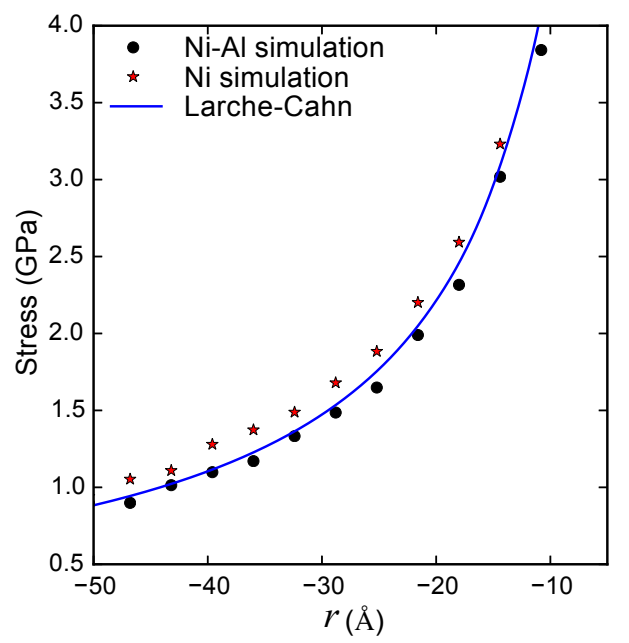

(b)

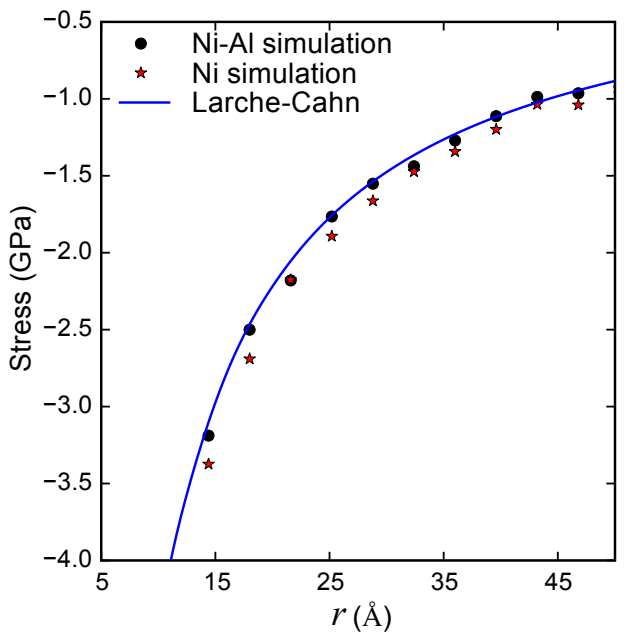

(c)

Figure 3: (a) Hydrostatic stress near the dislocation in the Ni-Al alloy at $700 \mathrm{~K}$ as a function of distance $r$ in vertical direction. (b) Zoom into the tension region. (c) Zoom into the compression region. 


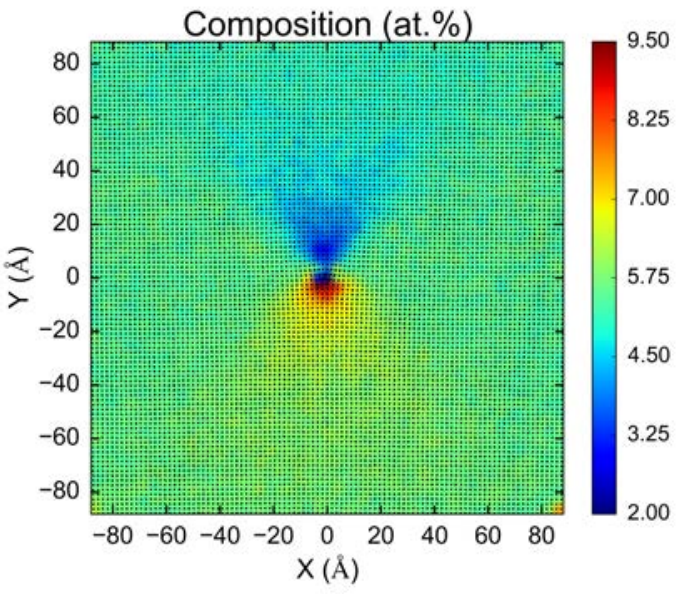

(a)

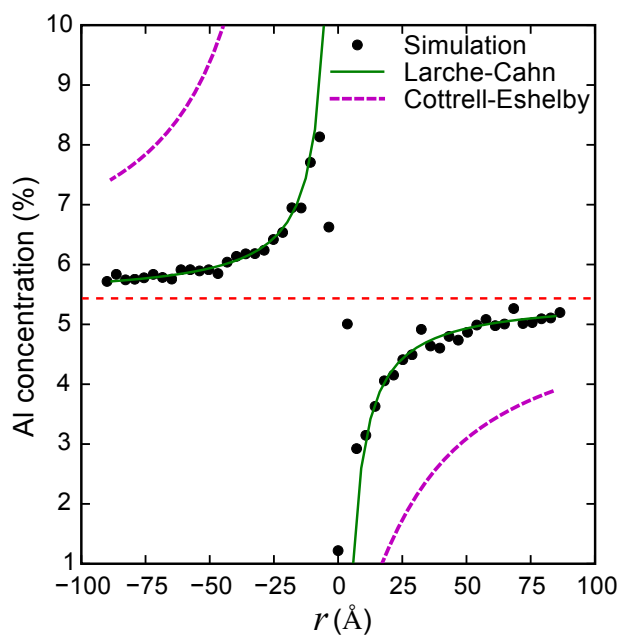

(b)

Figure 4: (a) Color map of $\mathrm{Al}$ concentration around the dislocation in the $\mathrm{Ni}-\mathrm{Al}$ alloy at $700 \mathrm{~K}$. (b) $\mathrm{Al}$ concentration near the dislocation in the $\mathrm{Ni}-\mathrm{Al}$ alloy as a function of distance $r$ in vertical direction. 
(a)
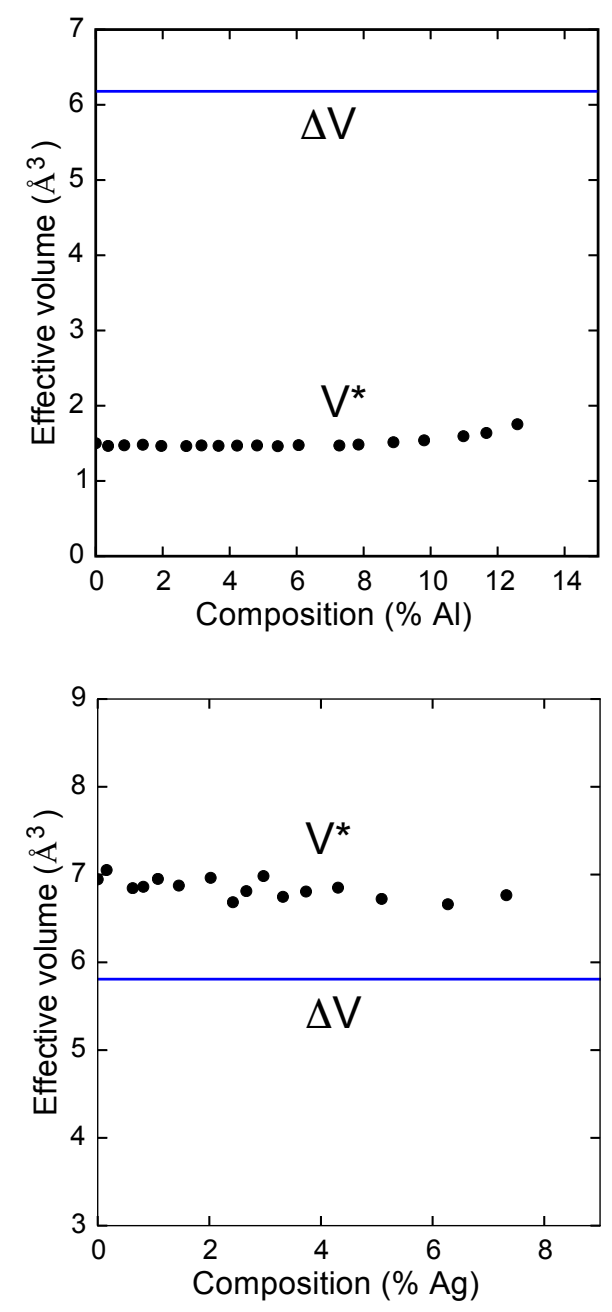

(b)

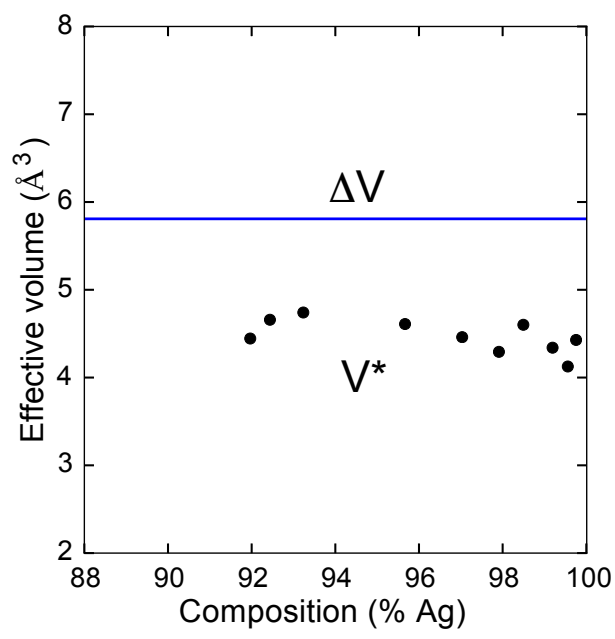

(c)

Figure 5: Comparison of the volume change per solute atom in the theories by Cottrell and Eshelby $(\Delta V)$ and Larché and Cahn $\left(V^{*}\right)$. The points represent simulation results for $V^{*}$ in the (a) gamma-phase of the $\mathrm{Ni}-\mathrm{Al}$ system, (b) $\mathrm{Cu}$-rich solid solution of the $\mathrm{Cu}-\mathrm{Ag}$ system, and (c) Ag-rich solid solution of the $\mathrm{Cu}-\mathrm{Ag}$ system. The simulation results end near the respective solubility limits. 


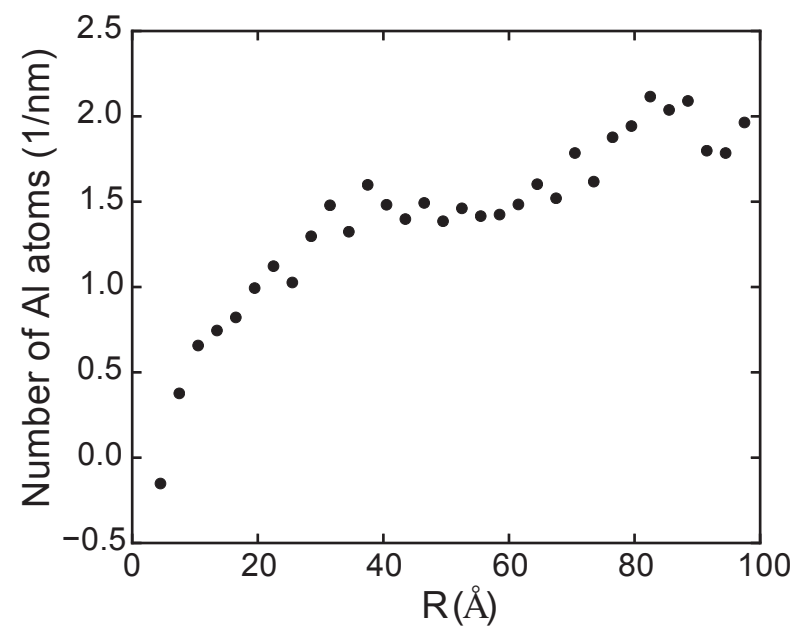

(a)

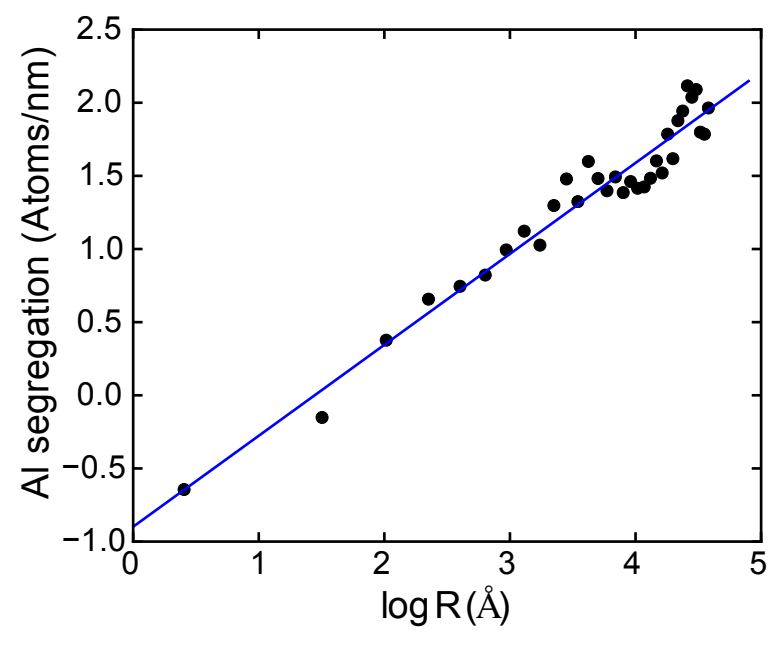

(b)

Figure 6: Total number $\Gamma$ of excess $\mathrm{Al}$ atoms in dislocation atmosphere per unit dislocation length in the $\mathrm{Ni}-\mathrm{Al}$ alloy at $700 \mathrm{~K}$. (a) $\Gamma$ as a function of the cylinder radius $R$. (b) $\Gamma$ as a function of $\log R$. The linear regression line is shown as a guide to the eye. 
Graphical Abstract
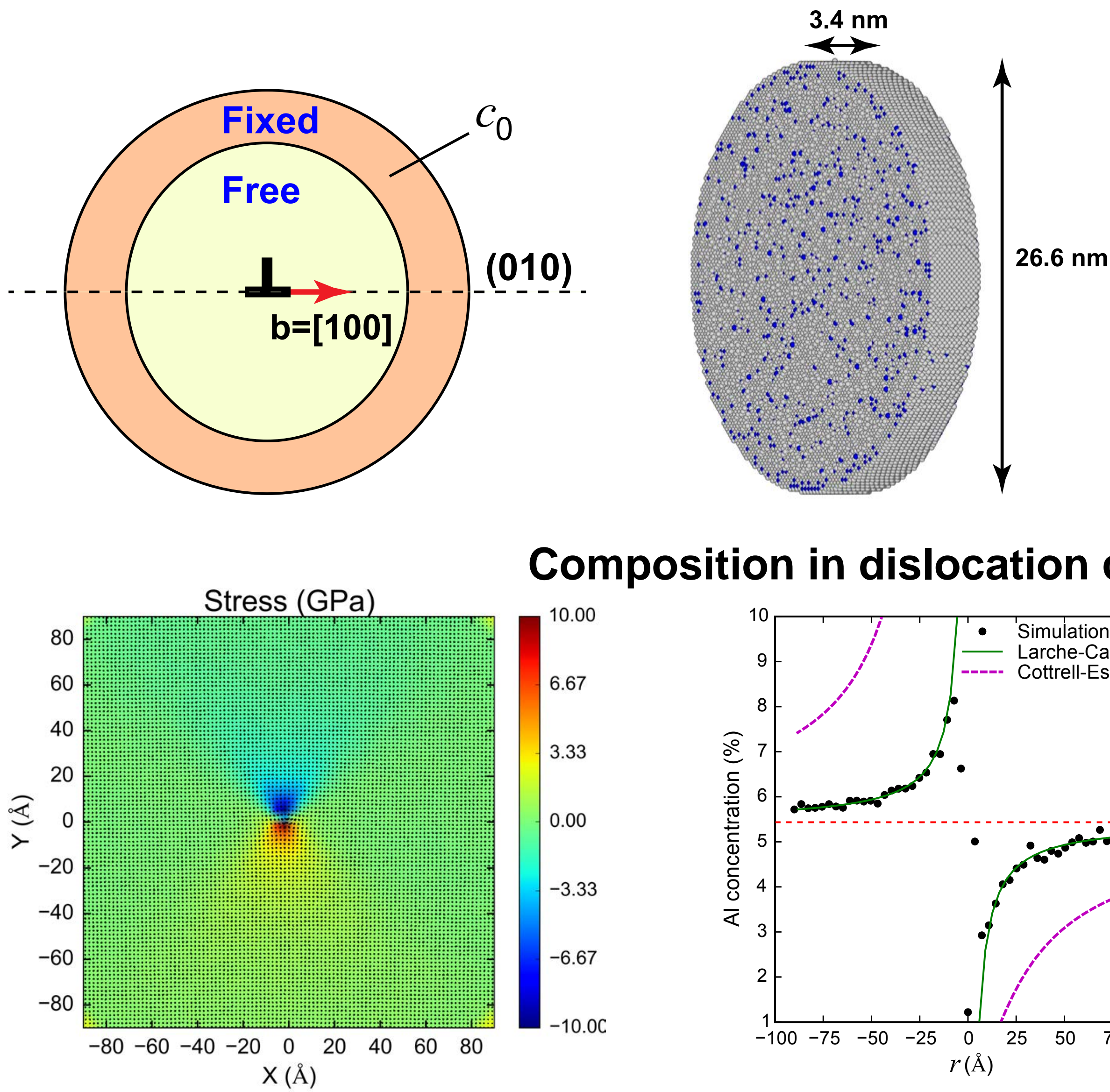

\section{Composition in dislocation core}

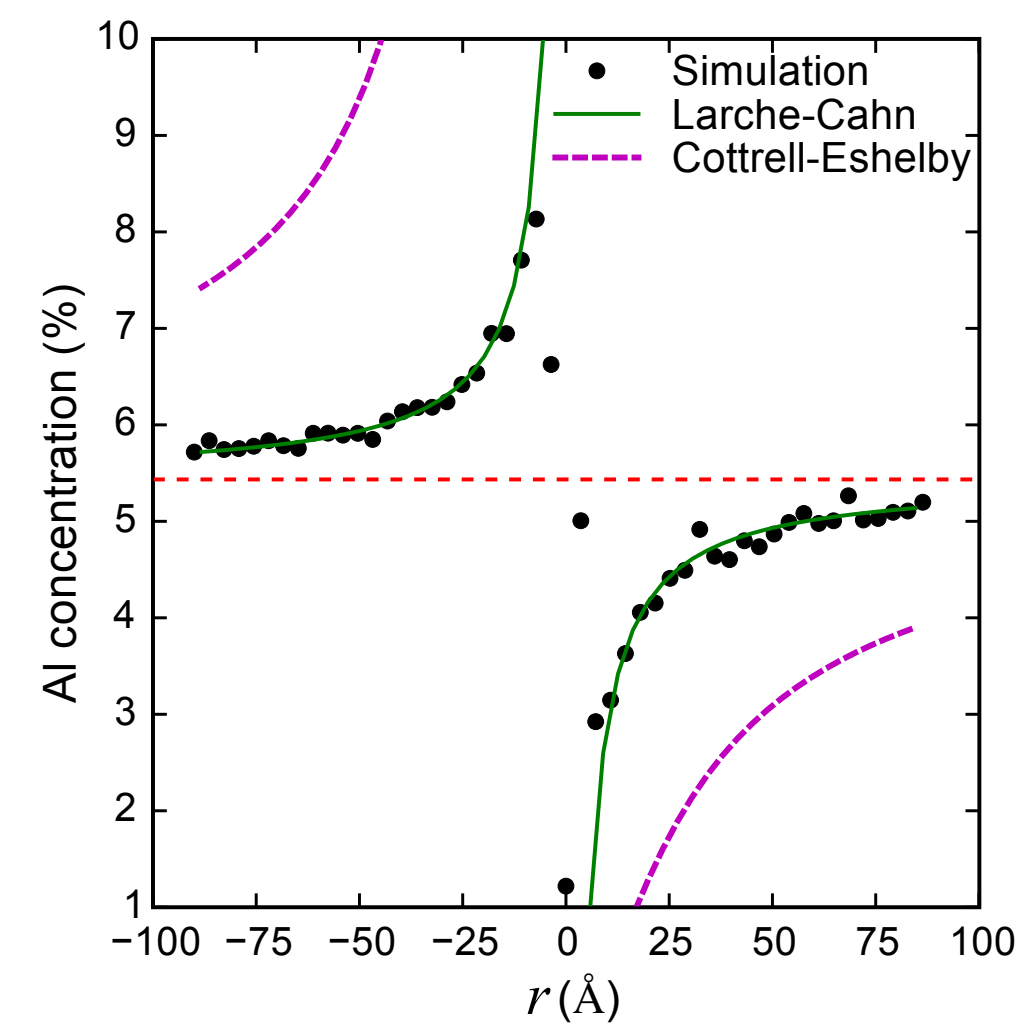

